\title{
Computing Tropical Varieties Over Fields with Valuation
}

\author{
Thomas Markwig ${ }^{1} \cdot$ Yue Ren ${ }^{2}$
}

Received: 20 January 2017 / Revised: 18 June 2018 / Accepted: 24 June 2019 / Published online: 5 August 2019 (c) The Author(s) 2019

\section{Abstract}

We show how the tropical variety of an ideal $I \unlhd K\left[x_{1}, \ldots, x_{n}\right]$ over a field $K$ with non-trivial discrete valuation can always be traced back to the tropical variety of an ideal $\pi^{-1} I \unlhd R \llbracket t \rrbracket\left[x_{1}, \ldots, x_{n}\right]$ over some dense subring $R$ in its ring of integers. We show that this connection is compatible with the Gröbner polyhedra covering them. Combined with previous works, we thus obtain a framework for computing tropical varieties over general fields with valuations, which relies on the existing theory of standard bases if $\pi^{-1} I$ is generated by elements in $R\left[t, x_{1}, \ldots, x_{n}\right]$.

Keywords Tropical geometry · Tropical varieties · Gröbner fans · Standard bases · Valued fields

Mathematics Subject Classification 14T05 $\cdot 13 \mathrm{P} 10 \cdot 13 \mathrm{~F} 25 \cdot 12 \mathrm{~J} 25$

Communicated by Joseph M. Landsberg.

The first and second authors were partially supported by the DFG transregional collaborative research centre SFB-TRR195 Symbolic Tools in Mathematics and their Application and the DFG priority project SPP1489 Algorithmic and Experimental Methods in Algebra, Geometry and Number Theory, respectively. Both authors would like to thank the Mittag-Leffler Institute for its hospitality during the program Tropical Geometry, Amoebas and Polytopes in spring 2018.

$\bowtie \quad$ Yue Ren

yue.ren@mis.mpg.de

https://www.yueren.de/

Thomas Markwig

keilen@math.uni-tuebingen.de

https://www.math.uni-tuebingen.de/ keilen

1 Eberhard Karls Universität Tübingen - Fachbereich Mathematik, Auf der Morgenstelle 10, 72076 Tübingen, Germany

2 Max Planck Institute for Mathematics in the Sciences, Inselstr. 22, 04103 Leipzig, Germany

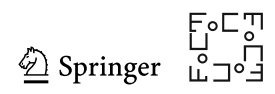




\section{Introduction}

Given a polynomial ideal $I$ over a field $K$ with a non-trivial valuation $v: K \rightarrow$ $\mathbb{R} \cup\{\infty\}$, its tropical variety $\mathcal{T}(I)$ is commonly described as the combinatorial shadow of its vanishing set over the algebraic closure of $K$. Tropical varieties arise naturally in many contexts in mathematics $[1,22]$ and beyond, such as phylogenetic trees in biology [24, §4], product-mix auctions in economics [2,32] or finiteness of central configurations in the 5-body problem in physics [12].

However, computing tropical varieties is an algorithmically highly challenging task, requiring sophisticated techniques from computer algebra and convex geometry. The first techniques were developed by Bogart, Jensen, Speyer, Sturmfels and Thomas [5] for the rational function field over the complex numbers $\mathbb{C}(t)$ using classical Gröbner basis methods. More recently, Chan and Maclagan [7] generalised the notion of Gröbner bases to general fields with valuation in order to compute tropical varieties thereover. The linchpin of both works is the ability to compute initial ideals. Moreover, significant advances have been made in specific parts of the computations: Chan [6], Hofmann and Ren [13], Sommars and Verschelde [29] all worked on improving the main bottleneck that is the computation of tropical links. The first two works developed new algorithms based on projections and intersections, respectively, whereas the latter improved the computation of so-called tropical prevarieties which was essential for the original algorithm. At the same time, Vaccon [34] showed that Matrix-F5 ideas can be applied to improve the performance of the generalised Gröbner bases computation.

In contrast, this article revisits the problem on a more fundamental level. As in [7], the overall goal is to develop a framework for general fields with valuation in which the original algorithms in [5] work almost ad verbum. However, instead of introducing a new notion of Gröbner bases, we aim to base it on the existing theory of standard bases [25]. The key idea is to use Cohen's Structure Theorem and replace the valued field $K$ with a power series ring $R \llbracket t \rrbracket$ with its natural valuation. This replaces the original ideal $I \unlhd K[x]$ with an ideal in $R \llbracket t \rrbracket[x]$, which is generated by polynomials in both $t$ and $x$ under mild assumptions on $I$. Our approach is to a certain extent equivalent to that of Chan and Maclagan, which can be seen from the fact that we naturally obtain an algorithm for computing their Gröbner bases. However, we can leverage existing implementations, such as in the computer algebra system SINGULAR [8], for a better performance (see Timings 1).

Our framework relies heavily on two previous works: In [19], we introduced standard bases for ideals in $R \llbracket t \rrbracket[x]$, whose elements are multivariate polynomials in $x$ and univariate power series in $t$ over a coefficient ring $R$. In [20], we introduced Gröbner fans for ideals in $R \llbracket t \rrbracket[x]$, which are a natural amalgamation of the existing notions of Gröbner fans for power series rings [3,26,33] and Gröbner fans for polynomial rings over coefficient rings [23]. In both works, special emphasis is put on ideals in $R \llbracket t \rrbracket[x]$ generated by polynomials in $t$ and $x$. For those, our standard bases coincide with the existing notion of standard bases for polynomials over coefficient rings and our algorithms consist of a finite sequence of basic polynomial arithmetic.

This article is organised as follows: First, in Sect. 2, we recall Cohen's Structure Theorem and use it to establish a bijection between the tropical variety of an ideal in $K[x]$ and the tropical variety of a corresponding ideal in $R \llbracket t \rrbracket[x]$ (see Theorem 4).

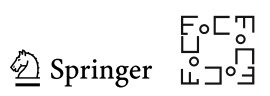


Next, in Sect. 3, we show that this bijection is compatible with the polyhedral structures covering the respective tropical varieties (see Corollary 3). Finally, in Sect. 4, we explain how the corresponding ideal in $R \llbracket t \rrbracket[x]$ can be computed from the original ideal in $K[x]$.

Furthermore, modified versions of the algorithms in [5] in our framework have been implemented in the SINGULAR library tropical.1ib [15], relying on GFANLIB $[14,16]$ for computations in convex geometry (see Example 5). They are publicly available as part of the official SINGULAR distribution, and a detailed account on the modified algorithms can be found in [27].

\section{Tracing Tropical Varieties to a Trivial Valuation}

The aim of this section is to show how tropical varieties over a valued field $K$ can be traced back to tropical varieties over a power series ring $R \llbracket t \rrbracket$ as in Convention 2 . The linchpin is to show how initial ideals over one can be described through initial ideals over the other, and the remaining results then follow naturally from this. Let us start by recalling Cohen's Structure Theorem.stop

Theorem 1 (Cohen's Structure Theorem, [21, §23 Corollary 5]) Let R be a Noetherian ring, $P=\langle p\rangle \unlhd R$ an ideal and $\widehat{R}$ the $P$-adic completion of $R$. Then

$$
\widehat{R} \cong R \llbracket t \rrbracket /\langle p-t\rangle,
$$

where $R \llbracket t \rrbracket$ denotes the ring of formal power series in $t$ with coefficients in $R$.

Convention 2 For the remainder of the article, fix a field $K$ with a non-trivial discrete valuation $v: K \rightarrow \mathbb{R} \cup\{\infty\}$. Without loss of generality, we may assume that $K$ is complete in the topology induced by the valuation, as in our context we can always pass to its completion if it is not. Let $\mathcal{O}_{K}$ be its ring of integers, and let $\mathfrak{K}$ denote its residue field. Fix a uniformising parameter $p \in \mathcal{O}_{K}$, and $R \leq \mathcal{O}_{K}$ a subring that is dense in the topology induced by the valuation, i.e. $\widehat{R}=\mathcal{O}_{K}$. By Theorem 1 , we have two exact sequences

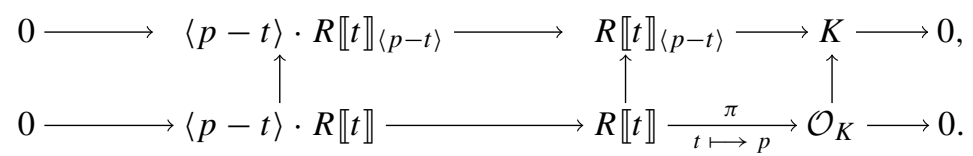

Moreover, fix a multivariate polynomial ring $K[x]=K\left[x_{1}, \ldots, x_{n}\right]$. By abuse of notation, we also use $\pi$ to refer to both the map $R \llbracket t \rrbracket[x] \rightarrow \mathcal{O}_{K}[x]$ as well as the composition $R \llbracket t \rrbracket[x] \rightarrow \mathcal{O}_{K}[x] \hookrightarrow K[x]$, where $R \llbracket t \rrbracket[x]$ and $\mathcal{O}_{K}[x]$ denote the rings of polynomials in $x_{1}, \ldots, x_{n}$ with coefficients in $R \llbracket t \rrbracket$ and $\mathcal{O}_{K}$, respectively.

Example 1 ( $p$-adic numbers) The most interesting example is the field $K:=\mathbb{Q}_{p}$ of $p$-adic numbers with $\mathcal{O}_{K}:=\mathbb{Z}_{p}$ the ring of $p$-adic integers. Then $R:=\mathbb{Z} \leq \mathbb{Z}_{p}$ is a natural dense subring, which is computationally easy to work with. The exact sequences in Convention 2 merely reflect the presentation of $p$-adic integers as power series in $p$ : 


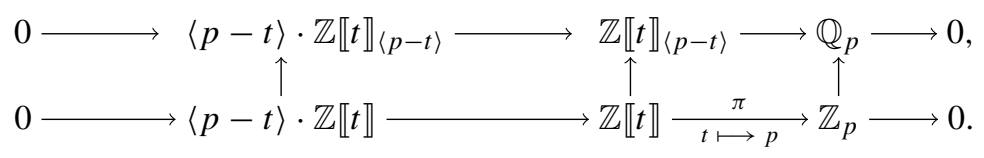

Example 2 Given the choice of $R \leq \mathcal{O}_{K}$ in Convention 2, choosing $R:=\mathcal{O}_{K}$ is always possible. However, in many examples there are natural choices for $R$, which are computationally much easier to handle than $\mathcal{O}_{K}$ itself:

1. $K=k((t))$ the field of Laurent series over a field $k$ with $\mathcal{O}_{K}=k \llbracket t \rrbracket$ the ring of power series over $k, R=k[t]$ and $p=t$; e.g. $k=\mathbb{F}_{q}$ with $q$ a prime power, as used in [30, Sect. 7] or [17], or $k=\mathbb{Q}$ as considered in [5] (see Example 4).

2. Finite extensions $K$ of $\mathbb{Q}_{p}$ and $\mathbb{F}_{q}((t))$, i.e. all local fields with non-trivial valuation, and also all higher-dimensional local fields.

3. $\mathcal{O}_{K}$ any completion of a localisation of a Dedekind domain $R$ at a prime ideal $P \unlhd R, p \in P$ a suitable element. Note that $p$ does not need to generate $P$ and hence $\mathcal{O}_{K}$ need not be the completion with respect to the ideal generated by $p$, e.g. $R=\mathbb{Z}[\sqrt{-5}], P=\langle 2,1+\sqrt{-5}\rangle$ and $p=2$.

4. For an odd choice of $R$, consider $K:=\mathbb{Q}(s)((t))$ so that $\mathcal{O}_{K}=\mathbb{Q}(s) \llbracket t \rrbracket$. Set $R:=S^{-1} \mathbb{Q}[s, t]$, where $S:=\mathbb{Q}[s, t] \backslash(\langle t-1, s\rangle \cup\langle t\rangle)$ is multiplicatively closed as the complement of two prime ideals. Then $R$ is a non-catenarian, dense subring of $\mathcal{O}_{K}$.

To fix the notation, we briefly recall some basic notions in tropical geometry that are of immediate relevance to us. For an in-depth introduction to tropical geometry, we refer to the reader to [18]. For a brief survey with a view towards algebraic geometry, we recommend [9].

Definition 1 (Initial forms, initial ideals, tropical varieties over valued fields) For a polynomial $0 \neq f=\sum_{\alpha \in \mathbb{N}^{n}} c_{\alpha} \cdot x^{\alpha} \in K[x]$ and a weight vector $w \in \mathbb{R}^{n}$, we define the valued weighted degree and initial form of $f$ with respect to $w$ to be:

$$
\begin{aligned}
\operatorname{deg}_{v, w}(f) & :=\max \left\{w \cdot \alpha-v\left(c_{\alpha}\right) \mid c_{\alpha} \neq 0\right\} \in \mathbb{R}, \\
\operatorname{in}_{v, w}(f) & :=\sum_{w \cdot \alpha-v\left(c_{\alpha}\right) \text { maximal }} \overline{c_{\alpha} \cdot p^{-v\left(c_{\alpha}\right)}} \cdot x^{\alpha} \in \mathfrak{K}[x] .
\end{aligned}
$$

For an ideal $I \unlhd K[x]$ and a weight vector $w \in \mathbb{R}^{n}$, we define the initial ideal of I with respect to $w$ to be:

$$
\operatorname{in}_{v, w}(I):=\left\langle\operatorname{in}_{v, w}(f) \mid 0 \neq f \in I\right\rangle \unlhd \mathfrak{K}[x] .
$$

We refer to the set of weight vectors for which the initial ideal contains no monomial as the tropical variety of $I$,

$$
\mathcal{T}_{v}(I):=\left\{w \in \mathbb{R}^{n} \mid \operatorname{in}_{v, w}(I) \text { monomial free }\right\}
$$

Theorem 3 (Structure Theorem for Tropical Varieties, [18, Theorem 3.3.5]) Let I $\unlhd$ $K[x]$ define an irreducible subvariety in $\left(K^{*}\right)^{n}$ of dimension $d$. Then $\mathcal{T}_{v}(I)$ is the

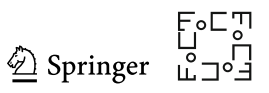


support of a pure polyhedral complex of the same dimension that is connected through codimension 1.

Next, we introduce initial forms and initial ideals in for elements and ideals in $R \llbracket t \rrbracket[x]$ and show how initial ideals of ideals in $R \llbracket t \rrbracket[x]$ can be used to compute the initial ideals of ideals in $K[x]$.

Definition 2 (Initial forms, initial ideals) Given an element $0 \neq f=\sum_{\beta, \alpha} c_{\alpha, \beta}$. $t^{\beta} x^{\alpha} \unlhd R \llbracket t \rrbracket[x]$ and a weight vector $w \in \mathbb{R}_{<0} \times \mathbb{R}^{n}$, we define the weighted degree and initial form of $f$ with respect to $w$ to be

$$
\begin{aligned}
\operatorname{deg}_{w}(f) & :=\max \left\{w \cdot(\beta, \alpha) \mid c_{\beta, \alpha} \neq 0\right\} \in \mathbb{R}, \\
\operatorname{in}_{w}(f) & :=\sum_{w \cdot(\beta, \alpha) \text { maximal }} c_{\alpha, \beta} \cdot t^{\beta} x^{\alpha} \in R[t, x],
\end{aligned}
$$

where $R[t, x]$ denotes the ring of polynomials in $t, x_{1}, \ldots, x_{n}$ with coefficients in $R$.

Given an ideal $I \unlhd R \llbracket t \rrbracket[x]$ and a weight vector $w \in \mathbb{R}_{<0} \times \mathbb{R}^{n}$, we define the initial ideal of $I$ with respect to $w$ to be:

$$
\operatorname{in}_{w}(J):=\left\langle\operatorname{in}_{w}(f) \mid 0 \neq f \in J\right\rangle \unlhd R[t, x] .
$$

This can be thought of as a natural extension of Definition 1 with trivial valuation on the coefficients. Note that we only allow weight vectors with negative weight in $t$, so that the maximum of all $w \cdot(\beta, \alpha)$ exists.

Example 3 ( $[6, \S 3.6])$ Consider, over the field of 3-adic numbers $\mathbb{Q}_{3}$, the ideal

$$
I=\left\langle 2 x_{1}^{2}+3 x_{1} x_{2}+24 x_{3} x_{4}, 8 x_{1}^{3}+x_{2} x_{3} x_{4}+18 x_{3}^{2} x_{4}\right\rangle \unlhd \mathbb{Q}_{3}\left[x_{1}, \ldots, x_{4}\right]=\mathbb{Q}_{3}[x],
$$

and the weight vector $w:=(1,11,3,19)$. The initial ideal of $I$ under the 3 -adic valuation is then

$$
\operatorname{in}_{v_{3}, w}(I)=\left\langle x_{1}^{2}, x_{1} x_{3} x_{4}, x_{1} x_{2}^{2} x_{3}, x_{1} x_{2}^{4}, x_{3}^{4} x_{4}^{2}\right\rangle \unlhd \mathbb{F}_{3}[x]
$$

Moreover, we have

$$
\pi^{-1} I=\left\langle 3-t, 2 x_{1}^{2}+3 x_{1} x_{2}+24 x_{3} x_{4}, 8 x_{1}^{3}+x_{2} x_{3} x_{4}+18 x_{3}^{2} x_{4}\right\rangle \unlhd \mathbb{Z} \llbracket t \rrbracket[x],
$$

and for the weight vector $(-1, w) \in \mathbb{R}_{<0} \times \mathbb{R}^{4}$ a short standard bases computation (see Proposition 3) yields

$$
\operatorname{in}_{(-1, w)}\left(\pi^{-1} I\right)=\left\langle 3, x_{1}^{2}, t x_{1} x_{3} x_{4}, t^{3} x_{1} x_{2}^{2} x_{3}, t^{4} x_{1} x_{2}^{4}, t^{3} x_{3}^{4} x_{4}^{2}\right\rangle \unlhd \mathbb{Z}[t, x] .
$$

The similarity to the initial ideal of $I$ under the 3 -adic valuation is no coincidence.

Proposition 1 For any ideal $I \unlhd \mathcal{O}_{K}[x]$ and any weight vector $w \in \mathbb{R}^{n}$, we have:

$$
\left.\overline{\operatorname{in}}(-1, w)_{\left(\pi^{-1} I\right) \mid}\right|_{t=1}=\operatorname{in}_{v, w}(I)
$$


where $\overline{(\cdot)}$ denotes the canonical projection $\overline{(\cdot)}: R[x] \rightarrow \mathfrak{K}[x]$.

Proof $\supseteq$ Any term $s \in \mathcal{O}_{K}[x]$ is of the form $s=\left(\sum_{\beta} c_{\beta} p^{\beta}\right) \cdot x^{\alpha}$ with $p \nmid c_{\beta}$ for all $\beta \in \mathbb{N}$. Then the element $s^{\prime}:=\left(\sum_{\beta} c_{\beta} t^{\beta}\right) \cdot x^{\alpha} \in R \llbracket t \rrbracket[x]$ is a natural preimage of $s$ under $\pi$ for which we have

$$
\operatorname{in}_{\nu, w}(s)=\bar{c}_{\beta_{0}} \cdot x^{\alpha}={\overline{\operatorname{in}}(-1, w)\left(s^{\prime}\right) \mid}_{t=1} \text {, where } \beta_{0}=\min \left\{\beta \in \mathbb{N} \mid c_{\beta} \neq 0\right\} .
$$

And because the valued weighted degree of $s$ in $\mathcal{O}_{K}[x]$, i.e. the left-hand side in the following equation, and the weighted degree of $s^{\prime}$ in $R \llbracket t \rrbracket[x]$, i.e. the right-hand side of the following equation, coincide,

$\operatorname{deg}_{w}\left(x^{\alpha}\right)-v\left(\sum_{\beta} c_{\beta} p^{\beta}\right)=\max \left\{w \cdot \alpha-\beta \mid c_{\beta} \neq 0\right\}=\operatorname{deg}_{(-1, w)}\left(\sum_{\beta} c_{\beta} \cdot t^{\beta} x^{\alpha}\right)$,

this implies that any $f \in \mathcal{O}_{K}[x]$ has a preimage $f^{\prime} \in R \llbracket t \rrbracket[x]$ under $\pi$ such that

$$
\operatorname{in}_{v, w}(f)=\left.\overline{\operatorname{in}}(-1, w)\left(f^{\prime}\right)\right|_{t=1},
$$

simply by applying the above argument to each of its terms.

$\subseteq$ Once again consider a term $s=\sum_{\beta} c_{\beta} p^{\beta} \cdot x^{\alpha} \in \mathcal{O}_{K}[x]$ with $p \nmid c_{\beta}$ for all $\beta \in \mathbb{N}$. Then any preimage of it under $\pi$ is of the form $s^{\prime}=\sum_{\beta} c_{\beta} t^{\beta} x^{\alpha}+r$ for some $r \in\langle p-t\rangle$.

If $\operatorname{deg}_{(-1, w)}(r)>\operatorname{deg}_{(-1, w)}\left(\sum_{\beta} c_{\beta} t^{\beta} x^{\alpha}\right)$, we have

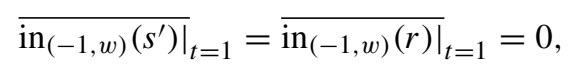

since $\operatorname{in}_{(-1, w)}(r) \in \operatorname{in}_{(-1, w)}\langle p-t\rangle=\langle p\rangle$.

And if $\operatorname{deg}_{(-1, w)}(r)<\operatorname{deg}_{(-1, w)}\left(\sum_{\beta} c_{\beta} t^{\beta} x^{\alpha}\right)$, we have

$$
\begin{aligned}
&{\overline{\left.\operatorname{in}_{(-1, w)}\left(s^{\prime}\right)\right|_{t=1}}}=\left.\overline{\operatorname{in}_{(-1, w)}\left(\sum_{\beta} c_{\beta} t^{\beta} x^{\alpha}\right)}\right|_{t=1}=\bar{c}_{\beta_{0}} \cdot x^{\alpha} \\
&=\operatorname{in}_{v, w}\left(\sum_{\beta} c_{\beta} p^{\beta} \cdot x^{\alpha}\right)=\operatorname{in}_{v, w}(s),
\end{aligned}
$$

where $\beta_{0}:=\min \left\{\beta \in \mathbb{N} \mid c_{\beta} \neq 0\right\}$.

Now suppose $\operatorname{deg}_{(-1, w)}(r)=\operatorname{deg}_{(-1, w)}\left(\sum_{\beta} c_{\beta} t^{\beta} x^{\alpha}\right)$. First observe that because $t$ is weighted negatively, there can be no cancellation amongst the highest weighted terms of $r$ and the terms of $\sum_{\beta} c_{\beta} t^{\beta} x^{\alpha}$, as the terms of $\sum_{\beta} c_{\beta} t^{\beta} x^{\alpha}$ are not divisible by $p$, unlike the terms of the highest weighted terms of $r$. Therefore, we have

$$
\overline{\left.\operatorname{in}_{(-1, w)}\left(s^{\prime}\right)\right|_{t=1}}=\underbrace{\left.\overline{\operatorname{in}_{(-1, w)}\left(\sum_{\beta} c_{\beta} t^{\beta} x^{\alpha}\right)}\right|_{t=1}}_{=\operatorname{in}_{v, w}\left(\sum_{\beta} c_{\beta} p^{\beta} \cdot x^{\alpha}\right)}+\underbrace{\overline{\operatorname{in}_{(-1, w)}(r) \mid}}_{=\overline{0}}=\operatorname{in}_{t=1}(s) .
$$




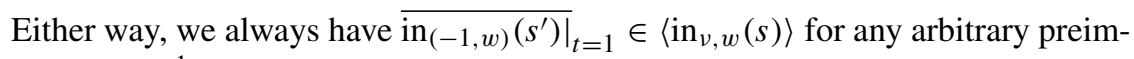
age $s^{\prime} \in \pi^{-1}(s)$, and, as before, the same hence holds true for any arbitrary element $f \in \mathcal{O}_{K}[x]$.

Corollary 1 For any ideal $I \unlhd K[x]$ and any weight vector $w \in \mathbb{R}^{n}$, we have:

$$
\left.\overline{\operatorname{in}(-1, w)}_{\left(\pi^{-1} I\right) \mid}\right|_{t=1}=\operatorname{in}_{v, w}(I) .
$$

Proof The statement follows from $\operatorname{in}_{v, w}(I)=\operatorname{in}_{v, w}\left(I \cap \mathcal{O}_{K}[x]\right)$ and Proposition 1.

Finally, we can introduce tropical varieties in $R \llbracket t \rrbracket[x]$ and show how they relate to tropical varieties in $K[x]$. In particular, we note how the tropical varieties in $R \llbracket t \rrbracket[x]$ that are of interest to us are pure and connected through codimension 1 . This is not a given for tropical varieties over coefficient rings $[18, \S 1.6]$ and very important algorithmically, as it allows us to run over it via a fan-traversal through the facets of the maximal cones.

Definition 3 (tropical variety) For an ideal $I \unlhd R \llbracket t \rrbracket[x]$, we define its tropical variety to be

$$
\mathcal{T}(I)=\operatorname{cl}\left(\left\{w \in \mathbb{R}_{<0} \times \mathbb{R}^{n} \mid \operatorname{in}_{w}(I) \text { monomial free }\right\}\right) \subseteq \mathbb{R}_{\leq 0} \times \mathbb{R}^{n},
$$

where $\mathrm{cl}(\cdot)$ denotes the closure in the Euclidean topology.

Theorem 4 Let $I \unlhd K[x]$ be an ideal. The projection $\mathbb{R}_{\leq 0} \times \mathbb{R}^{n} \rightarrow \mathbb{R}^{n}$ induces a bijection

$$
\begin{aligned}
\mathcal{T}\left(\pi^{-1} I\right) \cap\left(\{-1\} \times \mathbb{R}^{n}\right) & \stackrel{\sim}{\longrightarrow} \mathcal{T}_{v}(I) \\
\left(-1, w_{1}, \ldots, w_{n}\right) & \longmapsto\left(w_{1}, \ldots, w_{n}\right),
\end{aligned}
$$

where $v$ is the valuation on $K$ in Convention 2.

Proof For the bijection, we show that

$$
\operatorname{in}_{(-1, w)}\left(\pi^{-1} I\right) \text { is not monomial free } \Longleftrightarrow \operatorname{in}_{v, w}(I) \text { is not monomial free. }
$$

$\Longrightarrow$ Suppose $\operatorname{in}_{(-1, w)}\left(\pi^{-1} I\right) \unlhd R \llbracket t \rrbracket[x]$ contains a monomial $t^{\beta} x^{\alpha}$. By Corollary 1 , we have $\operatorname{in}_{v, w}(I)=\overline{\left.\operatorname{in}_{(-1, w)}\left(\pi^{-1} I\right)\right|_{t=1}}$, which means $\operatorname{in}_{v, w}(I)$ must contain the monomial $x^{\alpha} \in \mathfrak{K}[x]$.

$\Longleftarrow \operatorname{Suppose}_{\nu, w}(I) \unlhd \mathfrak{K}[x]$ contains a monomial $x^{\alpha}$. For the remainder of the proof, we abbreviate $(-1, w)$-weighted degree and $(-1, w)$-weighted homogeneous with weighted degree and weighted homogeneous, respectively. Consider all $r \in R[t, x]$ such that

$$
f:=t^{\beta} \cdot\left(x^{\alpha}+(t-1) \cdot r\right) \in \operatorname{in}_{(-1, w)}\left(\pi^{-1} I\right), \text { for some } \beta \in \mathbb{N} \text {. }
$$


Such $f$ exist, because, by Corollary 1 , in $(-1, w)\left(\pi^{-1} I\right)$ must contain an element of the form $x^{\alpha}+(t-1) \cdot r+p \cdot s$ for some $r, s \in R[t, x]$, and $p$ lies in $\operatorname{in}_{(-1, w)}\left(\pi^{-1} I\right)$. We may decompose each $r$ into its weighted homogeneous layers,

$$
r=r_{q_{1}}+\ldots+r_{q_{l}}
$$

with $\operatorname{deg}_{(-1, w)}\left(r_{q_{i}}\right)=q_{i}$ and $q_{i}<q_{i+1}$, and we may choose $\beta \in \mathbb{N}$ and $r \in R[t, x]$ such that $l$ is minimal. We now use the weighted homogeneity of $\operatorname{in}_{(-1, w)}\left(\pi^{-1} I\right)$ to show that $l=0$, which means that $\operatorname{in}_{(-1, w)}\left(\pi^{-1} I\right)$ contains the monomial $t^{\beta} x^{\alpha}$ for some $\beta \in \mathbb{N}$.

Assume $l>0$. Setting $d:=\operatorname{deg}_{(-1, w)}\left(x^{\alpha}\right)$ and $r_{q}=0$ for $q \notin\left\{q_{1}, \ldots, q_{l}\right\}$, we obtain the following weighted homogeneous layers of $f$ in weighted degree $q-\beta$ :

$$
f_{q-\beta}:= \begin{cases}t^{\beta} \cdot\left(t \cdot r_{q+1}-r_{q}\right), & \text { if } q \neq d \\ t^{\beta} \cdot\left(x^{\alpha}+t \cdot r_{d+1}-r_{d}\right), & \text { if } q=d .\end{cases}
$$

Since $\operatorname{in}_{(-1, w)}\left(\pi^{-1} I\right)$ is weighted homogeneous, all $f_{q-\beta}$ are contained in this ideal.

Now if $q_{l}$ was strictly bigger than $d$, we would get $f_{q_{l}-\beta}=-t^{\beta} \cdot r_{q_{l}} \in$ $\operatorname{in}_{(-1, w)}\left(\pi^{-1} I\right)$, and thus

$$
t^{\beta} \cdot\left(x^{\alpha}+(t-1) \cdot\left(r_{q_{1}}+\ldots+r_{q_{l-1}}\right)\right)=f-t^{\beta}(t-1) \cdot r_{q_{l}} \in \operatorname{in}_{(-1, w)}\left(\pi^{-1} I\right),
$$

contradicting our choice of $\beta$ and $r$ with minimal $l$.

Similarly, if $q_{1}$ was less than or equal to $d$, we would get $f_{q_{1}-1-\beta}=t^{\beta} \cdot t \cdot r_{q_{1}} \in$ $\operatorname{in}_{(-1, w)}\left(\pi^{-1} I\right)$, and thus

$$
t^{\beta+1} \cdot\left(x^{\alpha}+(t-1) \cdot\left(r_{q_{2}}+\ldots+r_{q_{l}}\right)\right)=t \cdot f-t^{\beta+1}(t-1) \cdot r_{q_{1}} \in \operatorname{in}_{(-1, w)}\left(\pi^{-1} I\right),
$$

again contradicting our choice of $\beta$ and $r$ with minimal $l$.

Hence, $q_{l} \leq d<q_{1}$, which, however, contradicts $q_{i}<q_{i+1}$.

Corollary 2 If $I \unlhd K[x]$ defines an irreducible subvariety of $\left(K^{*}\right)^{n}$ of dimension $d$, then $\mathcal{T}\left(\pi^{-1} I\right)$ is the support of a pure polyhedral fan of dimension $d+1$ connected through codimension one.

Proof Follows immediately from Definition 3 and Theorem 4, which imply that $\mathcal{T}\left(\pi^{-1} I\right)$ is the polyhedral fan over $\mathcal{T}_{v}(I)$. And by Theorem 3, the latter is pure of dimension $d$ and connected through codimension one.

We close the section with a couple of examples of tropical varieties over $K[x]$, their counterparts in $R \llbracket t \rrbracket[x]$ and how they can be computed in SINGULAR.

Example 4 Let $K:=\mathbb{Q}((\varepsilon))$ be the field of Laurent series, equipped with is natural valuation $v$, and let $I \unlhd K[x, y]$ be the principal ideal generated by $(x+y+1) \cdot\left(\varepsilon^{2} x+\right.$ $y+\varepsilon)$. Then $\mathcal{T}_{v}(I)$ is the union of two tropical lines, one with vertex at $(0,0)$ and one with vertex at $(1,-1)$. Setting $R:=\mathbb{Q}[t] \subseteq \mathcal{O}_{K}=\mathbb{Q} \llbracket t \rrbracket$, Theorem 4 implies that for

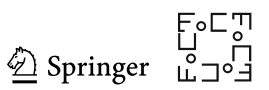




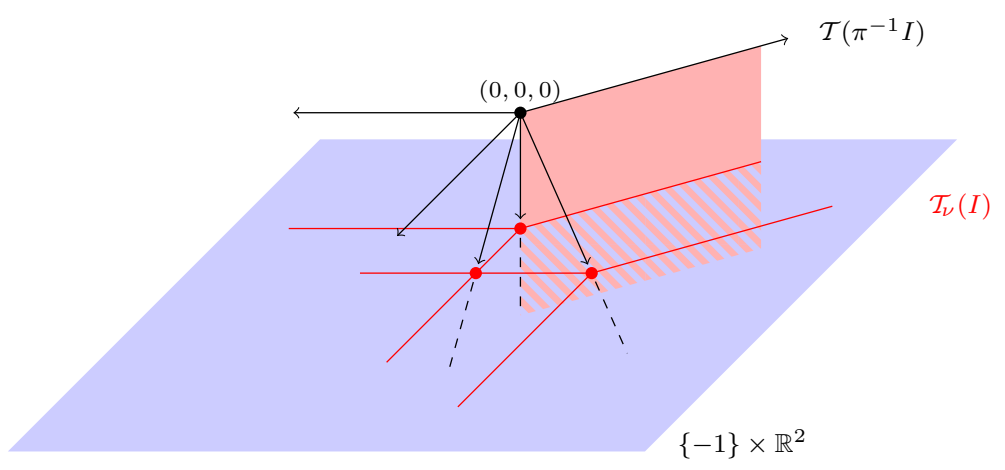

Fig. $1 \mathcal{T}\left(\pi^{-1} I\right)$ as polyhedral fan over $\mathcal{T}_{\mathcal{V}}(I)$

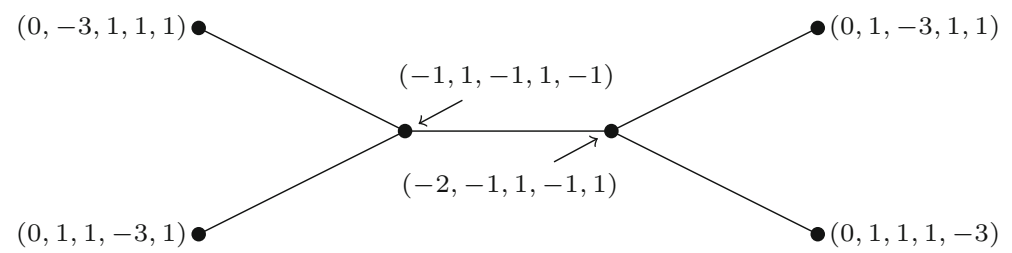

Fig. $2 \mathcal{T}\left(\left\langle x_{1}-2 x_{2}+3 x_{3}, 3 x_{2}-4 x_{3}+5 x_{4}, 2-t\right\rangle\right)$

any weight vector $w=\left(w_{t}, w_{x}, w_{y}\right) \in \mathbb{R}_{<0} \times \mathbb{R}^{2}$ in the lower open half-space we have

$$
w \in \mathcal{T}\left(\pi^{-1} I\right) \Longleftrightarrow\left(\frac{w_{x}}{\left|w_{t}\right|}, \frac{w_{y}}{\left|w_{t}\right|}\right) \in \mathcal{T}_{\nu}(I) .
$$

Hence $\mathcal{T}\left(\pi^{-1} I\right)$ is the polyhedral fan over $\mathcal{T}_{v}(I)$ as shown in Fig. 1. It consists of 6 rays and 8 two-dimensional cones in a way that the intersection with the affine hyperplane yields $\mathcal{T}_{v}(I)$.

Example 5 ([6, §3.6]) Consider $I:=\left\langle x_{1}-2 x_{2}+3 x_{3}, 3 x_{2}-4 x_{3}+5 x_{4}\right\rangle \unlhd$ $\mathbb{Q}\left[x_{1}, \ldots, x_{4}\right] \subseteq \mathbb{Q}_{2}\left[x_{1}, \ldots, x_{4}\right]$, whose preimage is given by

$$
\pi^{-1} I=\left\langle x_{1}-2 x_{2}+3 x_{3}, 3 x_{2}-4 x_{3}+5 x_{4}, 2-t\right\rangle \unlhd \mathbb{Z} \llbracket t \rrbracket\left[x_{1}, \ldots, x_{4}\right] .
$$

The tropical variety of the preimage is combinatorially of the form shown in Fig. 2 and invariant by translation under the one-dimensional subspace generated by $(0,1,1,1,1)$. Hence, each of the six drawn vertices represents a two-dimensional cone and each of the five edges represents a three-dimensional cone.

Intersecting with the affine hyperplane $\{-1\} \times \mathbb{R}^{4}$, we obtain a polyhedral complex as shown in the top left of Fig. 3, the vertices of Fig. 2 in $\{0\} \times \mathbb{R}^{4}$ becoming points at infinity. 

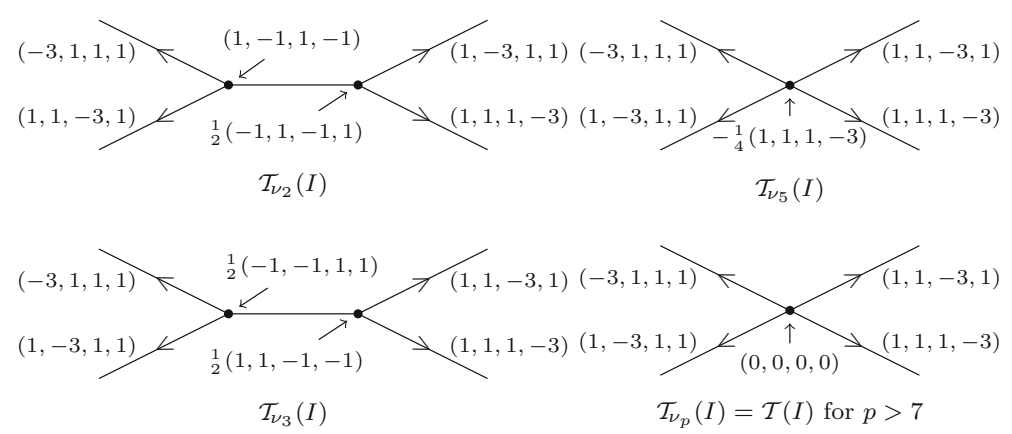

Fig. $3 \mathcal{T}_{v}(I)$ for all $p$-adic valuations and the trivial valuation

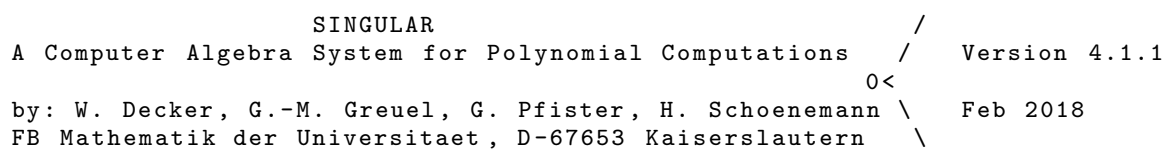

$>$ LIB "tropical.lib";

$>$ ring $r=0, x(1 \ldots 4), d p$;

$>$ ideal $I=$

$\mathrm{x}(1)+2 * \mathrm{x}(2)-3 * \mathrm{x}(3)$,
$3 * \mathrm{x}(2)-4 * \mathrm{x}(3)+5 * \mathrm{x}(4) ;$

$>$ number $\mathrm{p}=2$;

$>\operatorname{tropicalVariety}(I, p)$;

RAYS

$\begin{array}{lllllllll}-2 & -1 & 1 & -1 & 1\end{array} \quad$ \# 0

$\begin{array}{lllllllll}-1 & 1 & -1 & 1 & -1 & \end{array}$

$\begin{array}{llllllllllll}0 & -3 & 1 & 1 & 1 & \end{array}$

$\begin{array}{llllllll}0 & 1 & -3 & 1 & 1 & \end{array}$

$\begin{array}{lllllllllllll}0 & 1 & 1 & -3 & 1 & \end{array}$

$\begin{array}{llllllll}0 & 1 & 1 & 1 & -3 & \text { \# } & 5\end{array}$

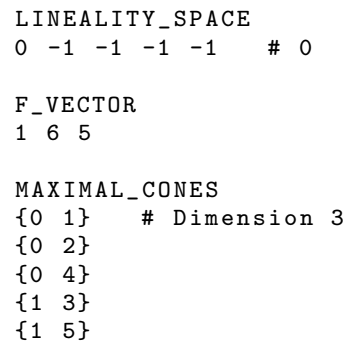

Fig. 4 Computing tropical varieties over fields with valuation in SINGULAR

Figure 3 further shows the tropical varieties of $I \unlhd \mathbb{Q}\left[x_{1}, \ldots, x_{4}\right]$ for other possible valuations on $\mathbb{Q}$. We see that regardless of the valuation, all tropical varieties share the same recession fan, as was proven by Gubler [11]. The latter is also necessarily the tropical variety under the trivial valuation. Note that for $p$ sufficiently large, the tropical varieties over $\mathbb{Q}_{p}$ coincide with the tropical variety under the trivial valuation. This is because $p$ is simply too large for $p-t$ to matter in any of our standard basis computations that arise in the computation of $\mathcal{T}\left(\pi^{-1} I\right)$. In the theory of modular Gröbner bases [4], these $p$ are referred to as good primes while the other $p$ are referred to as bad primes.

Figure 4 shows the input and output of the SINGULAR computation of $\mathcal{T}_{v_{2}}(I)$. Currently, the computation of tropical varieties is limited to the fields $\mathbb{C}\{\{t\}\}$ and $\mathbb{Q}_{p}$ and to ideals defined over $\mathbb{Q}$. 


\section{Tracing Gröbner Complexes to a Trivial Valuation}

In this section, we show how the Gröbner complexes of ideals in $K[x]$ can be traced back to the Gröbner fans of ideals in $R \llbracket t \rrbracket[x]$. We show how the latter induces a refinement of the former and how to determine whether two Gröbner cones map to the same Gröbner polyhedron. We close this section with a remark on $p$-adic Gröbner bases as introduced by Chan and Maclagan [7].

Definition 4 (Gröbner polyhedra, Gröbner complexes over valued fields) For a homogeneous ideal $I \unlhd K[x]$ and a weight vector $w \in \mathbb{R}^{n}$, we define its Gröbner polyhedra to be

$$
C_{v, w}(I):=\operatorname{cl}\left(\left\{v \in \mathbb{R}^{n} \mid \operatorname{in}_{v, v}(I)=\operatorname{in}_{v, w}(I)\right\}\right) \subseteq \mathbb{R}^{n}
$$

We refer to the collection $\Sigma_{v}(I):=\left\{C_{v, w}(I) \mid w \in \mathbb{R}^{n}\right\}$ as the Gröbner complex of $I$.

Theorem 5 ([18, Theorem 2.5.3]) Let $I \unlhd K[x]$ be a homogeneous ideal. Then all $C_{\nu, w}(I)$ are convex polytopes and $\Sigma_{v}(I)$ is a finite polyhedral complex.

Definition 5 For an $x$-homogeneous ideal $I \unlhd R \llbracket t \rrbracket[x]$, i.e. an ideal generated by elements which are homogeneous if considered as polynomials in $x$ with coefficients in $R \llbracket t \rrbracket$, and a weight vector $w \in \mathbb{R}_{<0} \times \mathbb{R}^{n}$ we define its Gröbner cone to be

$$
C_{w}(I):=\operatorname{cl}\left(\left\{v \in \mathbb{R}_{<0} \times \mathbb{R}^{n} \mid \operatorname{in}_{v}(I)=\operatorname{in}_{w}(I)\right\}\right) .
$$

We refer to the collection $\Sigma(I):=\left\{C_{w}(I), C_{w}(I) \cap\{0\} \times \mathbb{R}^{n} \mid w \in \mathbb{R}_{<0} \times \mathbb{R}^{n}\right\}$ as the Gröbner fan of $I$.

Proposition 2 ([20, Theorem 3.19]) Let $I \unlhd R \llbracket t \rrbracket[x]$ be an $x$-homogeneous ideal. Then all $C_{w}(I)$ are polyhedral cones and $\Sigma(I)$ is a finite polyhedral fan.

Corollary 3 The map $\{-1\} \times \mathbb{R}^{n} \stackrel{\sim}{\longrightarrow} \mathbb{R}^{n},(-1, w) \longmapsto w$ is compatible with the Gröbner fan $\Sigma\left(\pi^{-1} I\right)$ and the Gröbner complex $\Sigma_{v}(I)$, i.e. it maps the restriction of a Gröbner cone $C_{(-1, w)}\left(\pi^{-1} I\right) \cap\left(\{-1\} \times \mathbb{R}^{n}\right)$ into the Gröbner polytope $C_{\nu, w}(I)$.

Proof Follows directly from Proposition 1, as two weight vectors with the same initial ideal of $\pi^{-1} I \unlhd R \llbracket t \rrbracket[x]$ yield the same initial ideal of $I \unlhd K[x]$.

Note that it may happen that several cones are mapped into the same Gröbner polytope, i.e. that the image of the restricted Gröbner fan is a refinement of the Gröbner complex (see Example 6).

We now recall the notion of initially reduced standard bases of ideals in $R \llbracket t \rrbracket[x]$ from [19] and how they determine the inequalities and equations of Gröbner cones as shown in [20]. We then use these to decide whether two Gröbner cones are mapped to the same Gröbner polytope and, by doing so, show that no separate standard basis computation is required for this. 
Definition 6 (initially reduced standard bases) Fix the lexicographical ordering $>$ such that $x_{1}>\ldots>x_{n}>1>t$. Given a weight vector $w \in \mathbb{R}_{<0} \times \mathbb{R}^{n}$, we define the weighted ordering $>_{w}$ to be

$$
\begin{aligned}
t^{\beta} x^{\alpha}>_{w} t^{\delta} x^{\gamma}: \Longleftrightarrow & w \cdot(\beta, \alpha)>w \cdot(\delta, \gamma) \text { or } \\
& w \cdot(\beta, \alpha)=w \cdot(\delta, \gamma) \text { and } t^{\beta} x^{\alpha}>t^{\delta} x^{\gamma} .
\end{aligned}
$$

For $g \in R \llbracket t \rrbracket[x]$, the leading term $\operatorname{LT}_{>_{w}}(g)$ is the unique term of $g$ with maximal monomial under $>_{w}$ and for $I \unlhd R \llbracket t \rrbracket[x]$, the leading ideal $\mathrm{LT}_{>_{w}}(I)$ is the ideal generated by the leading terms of all its elements. A finite subset $G \subseteq I$ is called a standard basis of $I$ with respect to $>_{w}$, if the leading terms of its elements generate $\mathrm{LT}_{>}(I)$.

Suppose $G=\left\{g_{1}, \ldots, g_{k}\right\}$ with $g_{i}=\sum_{\alpha \in \mathbb{N}^{n}} g_{i, \alpha} \cdot x^{\alpha}, g_{i, \alpha} \in R \llbracket t \rrbracket$. We call $G$ initially reduced, if the set

$$
G^{\prime}:=\left\{\sum_{\alpha \in \mathbb{N}} \operatorname{LT}_{>}\left(g_{i, \alpha}\right) \cdot x^{\alpha} \mid i=1, \ldots, k\right\} \subseteq R[t, x],
$$

is reduced in the classical sense, i.e. no term in the tail of an element of $G^{\prime}$ is in the ideal generated by the leading terms of the elements in $G^{\prime}$.

Proposition 3 ([20, Algorithm 4.6]) Let $I \unlhd R \llbracket t \rrbracket[x]$ be an $x$-homogeneous ideal and $w \in \mathbb{R}_{<0} \times \mathbb{R}^{n}$ a weight vector. Then an initially reduced standard basis $G$ of $I$ with respect to $>_{w}$ can be computed using a finite sequence of arithmetic operations in $R \llbracket t \rrbracket[x]$. Moreover, if $I$ is generated by elements in $R[t, x]$, then it can be computed using a finite sequence of arithmetic operations in $R[t, x]$.

Proposition 4 ([20, Proposition 3.8, 3.11]) Let $I \unlhd R \llbracket t \rrbracket[x]$ be an $x$-homogeneous ideal, let $w \in \mathbb{R}_{<0} \times \mathbb{R}^{n}$ be a weight vector and let $G$ an initially reduced standard basis of I with respect to $>_{w}$. Then $\left\{\operatorname{in}_{w}(g) \mid g \in G\right\}$ is an initially reduced standard basis of $\operatorname{in}_{w}(I)$ with respect to $>_{w}$, and the Gröbner cone of I around $w$ is given by

$$
C_{w}(I)=\operatorname{cl}\left(\left\{v \in \mathbb{R}_{<0} \times \mathbb{R}^{n} \mid \operatorname{in}_{v}(g)=\operatorname{in}_{w}(g) \text { for all } g \in G\right\}\right) .
$$

We now show that our standard bases of $\pi^{-1} I \unlhd R \llbracket t \rrbracket[x]$ yield Gröbner bases of initial ideals of $I \unlhd K[x]$, allowing us to immediately decide whether two Gröbner cones of the former are mapped to the same Gröbner polytope of the latter.

Corollary 4 Let $I \unlhd K[x]$ be a homogeneous ideal, let $w \in \mathbb{R}^{n}$ be a weight vector and let $G$ be an initially reduced standard basis of $\pi^{-1} I$ with respect to the weighted ordering $>_{(-1, w)}$. Then

$$
\left\{\left.\overline{\operatorname{in}_{(-1, w)}(g)}\right|_{t=1} \mid g \in G\right\}
$$

is a standard basis of $\operatorname{in}_{v, w}(I)$ with respect to the fixed lexicographical ordering $>$ restricted to monomials in $x$.

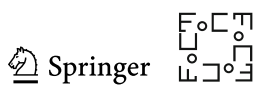


Proof By Proposition 4, the set $\operatorname{in}_{(-1, w)}(G):=\left\{\operatorname{in}_{(-1, w)}(g) \mid g \in G\right\}$ is an initially reduced standard basis of $\operatorname{in}_{(-1, w)}\left(\pi^{-1} I\right)$ with respect to $>_{(-1, w)}$. And because it is homogeneous with respect to the weight vector $(-1, w)$, it is also an initially reduced standard basis with respect to $>$. By choice of $>$, the set $\left.\operatorname{in}_{(-1, w)}(G)\right|_{t=1}$ remains a standard basis of $\left.\operatorname{in}_{(-1, w)}\left(\pi^{-1} I\right)\right|_{t=1}$ with respect to the restriction of $>$ to monomials in $x$. And since $\left.p \in \operatorname{in}_{(-1, w)}(G)\right|_{t=1},{\overline{\operatorname{in}_{(-1, w)}(G) \mid}}_{t=1}$ is a standard basis of $\overline{\left.\operatorname{in}_{(-1, w)}\left(\pi^{-1} I\right)\right|_{t=1}}$ with respect to the restriction of $>$.

Example 6 Consider the preimage $\pi^{-1} I \unlhd \mathbb{Z} \llbracket t \rrbracket[x, y, z]$ of the ideal $I=\left\langle 2 y+x, z^{2}+\right.$ $\left.y^{2}\right\rangle \unlhd \mathbb{Q}_{2}[x, y, z]$ and the two weight vectors $w=(1,3,7), v=(1,10,5) \in \mathbb{R}^{3}$. Fix a lexicographical tiebreaker $>$ with $x>y>z>1>t$.

The initially reduced standard basis of $\pi^{-1} I$ with respect to $>_{(-1, w)}$ and $>_{(-1, v)}$ is the following two sets, respectively (initial forms underlined):

$$
\begin{aligned}
G_{(-1, w)} & =\left\{\underline{2}-t, \underline{t y}+x, \underline{z^{2}}+y^{2}\right\}, G_{(-1, v)} \\
& =\left\{\underline{2}-t, \underline{t y}+x, \underline{x y}-t z^{2}, \underline{t^{2} z^{2}}+x^{2}, \underline{y^{2}}+z^{2}\right\},
\end{aligned}
$$

yielding the following Gröbner basis of $\operatorname{in}_{v, w}(I)$ and $\operatorname{in}_{v, v}(I)$ under $>$ :

$$
G_{\nu, w}=\left\{y, z^{2}\right\}, \quad G_{\nu, v}=\left\{y, x y, z^{2}, y^{2}\right\} .
$$

One immediately sees that both initial ideals coincide, meaning that the two Gröbner cones $C_{(-1, w)}\left(\pi^{-1} I\right)$ and $C_{(-1, v)}\left(\pi^{-1} I\right)$ are mapped to the same Gröbner polytope $C_{\nu_{2}, w}(I)=C_{\nu_{2}, v}(I)$.

Remark 1 ( p-adic Gröbner bases) By [18, Sect. 2.4], a Gröbner basis of an ideal $I \unlhd K[x]$ over valued fields with respect to a weight vector $w \in \mathbb{R}^{n}$ is a finite generating set whose initial forms generate the initial ideal $\operatorname{in}_{v, w}(I)$. By Corollary 4 , $\pi(G)$ is such a Gröbner basis if $G \subseteq \pi^{-1} I \unlhd R \llbracket t \rrbracket[x]$ is a standard basis under the monomial ordering $>_{w}$.

Lines 1 to 6 in Fig. 5 illustrate the computation of a Gröbner bases over the 2adic numbers in SINGULAR: Line 3 creates the Katsura(4) ideal in $x_{1}, \ldots, x_{4}$, Line 5 homogenises it on $x_{0}$ and adds the generator $2-t$, and Line 6 computes its standard basis. Note that $\mathrm{d}$ s is a weighted ordering with weight vector $(-1, \ldots,-1,-1)$ which

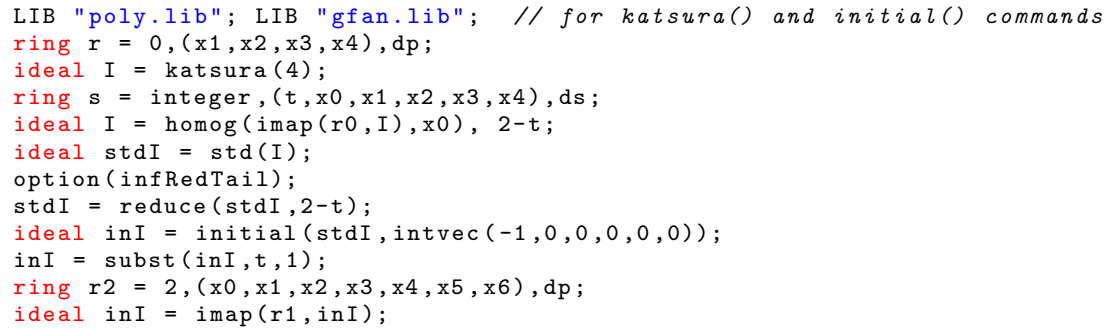

Fig. 5 Computing Gröbner bases over valued fields in SINGULAR 
Table 1 Timings in seconds unless aborted after $1 \mathrm{CPU}$ day

\begin{tabular}{lllll}
\hline Examples & GFAN & MACAULAY2 & SAGE & SINGULAR \\
\hline Cyclic(4) & - & 1 & 10 & 1 \\
Cyclic(5) & - & - & - & 1 \\
Cyclic(6) & - & - & - & 2 \\
Katsura(3) & 1 & 1 & 1 & 1 \\
Katsura(4) & - & - & 10 & 1 \\
Katsura(5) & - & - & 190 & 1 \\
Katsura(6) & - & - & 2900 & - \\
Chan & 1 & 1 & 4 & - \\
\hline
\end{tabular}

is equivalent to a weighted ordering with weight vector $(0, \ldots, 0,-1)$ since the ideal is homogeneous in $x_{0}, \ldots, x_{4}$. Substituting $t$ with 2 in staI yields a Gröbner basis, however the monomials will be out of order since the ordering ignores the 2-adic valuation.

Lines 7 to 12 in Fig. 5 construct the initial ideal: Line 7 forces SINGULAR to do tail reductions even though this might cause infinite loops in non-global orderings. Line 8 reduces staI with respect to $2-t$, so that the minimal degrees in $t$ reflect the 2 -valuations. Line 9 computes the desired initial form, and Line 10 replaces all $t$ with 1 so that Line 12 can safely pass to a polynomial ring without $t$ over the residue field.

Table 1 shows timings of the GFAN command gfan_padic -groebnerBasis by Anders Jensen [14], the MACAULAY2 Package GroebnerValuations by Andrew Chan [7,10], an implementation of a $p$-adic Matrix-F5 algorithm by Tris$\tan$ Vaccon in SAGE [28,34], and the standard basis engine of SINGULAR over integers under mixed orderings [8]. We consider the following examples:

Cyclic(n) In $\mathbb{Q}_{2}\left[x_{0}, \ldots, x_{n}\right]$, the cyclic ideal in the variables $x_{1}, \ldots, x_{n}$, homogenised using the variable $x_{0}$, and weight vector $(0, \ldots, 0)$.

Katsura(n) In $\mathbb{Q}_{2}\left[x_{0}, \ldots, x_{n}\right]$, the Katsura ideal in the variables $x_{1}, \ldots, x_{n}$, homogenised using the variable $x_{0}$, and weight vector $(0, \ldots, 0)$.

Chan In $\mathbb{Q}_{3}\left[x_{0}, \ldots, x_{4}\right]$, the ideal $\left\langle 2 x_{1}^{2}+3 x_{1} x_{2}+24 x_{3} x_{4}, 8 x_{1}^{3}+x_{2} x_{3} x_{4}+18 x_{3}^{2} x_{4}\right\rangle$ and weight vector $(-1,-11,-3,-19)$ taken from $[6, \S 3.6]$.

All computations were done on a server running Gentoo-3.16.5 with Intel Xeon E52690 processors. Computations exceeding $1 \mathrm{CPU}$ day were aborted. Note that the computations in SAGE were done up to a finite precision of $p^{50}$ and that the correctness of the result could only be verified for the examples for which either GFAN, MACAULAY2 or SiNGULAR finished.

\section{Computing the Preimage}

This article was dedicated to show how $\mathcal{T}_{v}(I)$ can be computed via $\mathcal{T}\left(\pi^{-1} I\right)$, however until now we have not addressed how to determine the preimage $\pi^{-1} I$ in the first place.

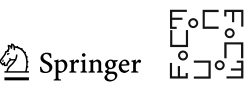


We therefore end the article with two results: The first shows that $\pi^{-1} I$ can be obtained by a saturation, and the second describes how to compute it.

Lemma 1 Let $I \unlhd K[x]$ be an ideal, and let $\left\{f_{1}, \ldots, f_{k}\right\} \subseteq I \cap \mathcal{O}_{K}[x]$ be a generating set over the valuation ring. Since $\pi: R \llbracket t \rrbracket[x] \rightarrow \mathcal{O}_{K}[x]$ is surjective, there are $f_{1}^{\prime}, \ldots, f_{k}^{\prime} \in R \llbracket t \rrbracket[x]$ such that $\pi\left(f_{i}^{\prime}\right)=f_{i} \in R[x]$. Then

$$
\pi^{-1} I=\left(\left\langle f_{1}^{\prime}, \ldots, f_{k}^{\prime}\right\rangle+\langle p-t\rangle\right): p^{\infty} \unlhd R \llbracket t \rrbracket[x]
$$

where $(\cdot): p^{\infty}$ denotes the saturation of the ideal with respect to $p$.

Proof The $\supseteq$ inclusion is obvious, as $p-t$ is mapped to 0 and $p$ is invertible in $K$.

For the converse inclusion, let $g \in \pi^{-1} I$. Then there are $q_{1}, \ldots, q_{k} \in K[x]$ such that

$$
\pi(g)=q_{1} \cdot f_{1}+\ldots+q_{k} \cdot f_{k} \in K[x]
$$

which means that for a sufficiently high power $l \in \mathbb{N}$ we have

$$
p^{l} \cdot \pi(g)=\underbrace{p^{l} q_{1}}_{\in \mathcal{O}_{K}[x]} \cdot f_{1}+\ldots+\underbrace{p^{l} q_{k}}_{\in \mathcal{O}_{K}[x]} \cdot f_{k} \in \mathcal{O}_{K}[x] .
$$

Since the map $\pi: R \llbracket t \rrbracket[x] \rightarrow \mathcal{O}_{K}[x]$ is surjective, there exist $q_{1}^{\prime}, \ldots, q_{k}^{\prime} \in R \llbracket t \rrbracket[x]$ such that

$$
p^{l} \cdot \pi(g)=\pi\left(q_{1}^{\prime} \cdot f_{1}^{\prime}+\ldots+q_{k}^{\prime} \cdot f_{k}^{\prime}\right),
$$

or rather

$$
p^{l} \cdot g-q_{1}^{\prime} \cdot f_{1}^{\prime}+\ldots+q_{k}^{\prime} \cdot f_{k}^{\prime} \in \operatorname{ker}(\pi)=\langle p-t\rangle
$$

Thus $p^{l} \cdot g \in\left\langle f_{1}^{\prime}, \ldots, f_{k}^{\prime}\right\rangle+\langle p-t\rangle$, and hence

$$
g \in\left(\left\langle f_{1}^{\prime}, \ldots, f_{k}^{\prime}\right\rangle+\langle p-t\rangle\right): p^{\infty}
$$

The next example shows that $\left\langle f_{1}, \ldots, f_{k}^{\prime}\right\rangle$ in Lemma 1 is not necessarily saturated with respect to $p$, which is why Proposition 5 shows how to compute it.

Example 7 Consider $I=\left\langle f_{1}, f_{2}\right\rangle \unlhd \mathbb{Q}_{2}[x, y]$, where $f_{1}=x^{2}+\frac{1}{2} y, f_{2}=y^{2}+\frac{1}{2} y$. Then $g=x^{2}-y^{2} \in I \cap \mathbb{Z}_{2}[x, y]$ and $2 g \in\left\langle f_{1}, f_{2}\right\rangle \unlhd \mathbb{Z}_{2}[x, y]$, but $g \notin\left\langle f_{1}, f_{2}\right\rangle \unlhd$ $\mathbb{Z}_{2}[x, y]$.

Lemma 2 Given the same conditions as in Lemma 1, we have

$$
\pi^{-1} I=\left(\left\langle f_{1}^{\prime}, \ldots, f_{k}^{\prime}\right\rangle+\langle p-t\rangle\right): t^{\infty} \unlhd R \llbracket t \rrbracket[x]
$$


Proof Follows directly from Lemma 1 , since $p-t \in\left\langle f_{1}^{\prime}, \ldots, f_{k}^{\prime}\right\rangle+\langle p-t\rangle$ implies that its saturation with respect to $p$ coincides with the saturation at $t$.

The following proposition shows how to compute the a standard basis of the preimage. It requires the notion of strong standard bases as in [25, Definition A.1.1.8]. The result and its proof is a straightforward generalisation of [31, Lemma 12.1].

Proposition 5 Let $I \unlhd K[x]$ be an ideal, and let $\left\{f_{1}, \ldots, f_{k}\right\} \subseteq I \cap \mathcal{O}_{K}[x]$ be a generating set of $I$ in the valuation ring. Since $\pi: R \llbracket t \rrbracket[x] \rightarrow \mathcal{O}_{K}[x]$ is surjective, there are $f_{1}^{\prime}, \ldots, f_{k}^{\prime} \in R \llbracket t \rrbracket[x]$ such that $\pi\left(f_{i}^{\prime}\right)=f_{i} \in \mathcal{O}_{K}[x]$.

Let $G \subseteq\left\langle f_{1}^{\prime}, \ldots, f_{k}^{\prime}\right\rangle+\langle p-t\rangle$ be a strong standard basis with respect to some weighted ordering $>_{w}$ with weight vector $w:=(-1,0, \ldots, 0)$. Then

$$
\left\{\frac{g}{t^{k}} \mid g \in G, t^{k} \text { highest power of } t \text { dividing } g\right\}
$$

is a strong standard basis of $\pi^{-1} I$ with respect to $>_{w}$.

Proof By Lemma 2, we have $G \subseteq \pi^{-1} I$. It remains to show that for any $f \in \pi^{-1} I$ there exists a $g \in G$ such that $\operatorname{LM}_{>_{w}}(g)$ divides $\operatorname{LM}_{>_{w}}(f)$. For that, observe that for any $f \in R \llbracket t \rrbracket[x]$ our ordering satisfies

$$
t \text { divides } \mathrm{LM}_{>w}(f) \Longleftrightarrow t \text { divides } f \text {. }
$$

Let $f \in \pi^{-1} I$. By Lemma $2, t^{l} f \in\left\langle f_{1}^{\prime}, \ldots, f_{k}^{\prime}\right\rangle+\langle p-t\rangle$ for $l \in \mathbb{N}$ sufficiently big. Since $G$ is strong, there is a $g \in G$ such that $\operatorname{LM}_{>}(g)$ divides $\operatorname{LM}_{>w}\left(t^{l} f\right)$. Let $t^{k}$ be the highest power of $t$ dividing $g$, so that $g / t^{k} \in G$. Then $\mathrm{LM}_{>_{w}}\left(g / t^{k}\right)$ has no common divisor with $t^{l}$, as by our first observation that would contradict the maximality of $k$. Hence it divides $\mathrm{LM}_{>}(f)$.

Example 8 Let $I$ be the ideal Katsura(4) from Remark 1. Figure 6 shows the initially reduced standard basis of the computation in Fig. 5. By Proposition 5, dividing stdI [6] and staI [7] by $t$ yields a standard basis of the preimage. This shows that stdI does not generate the entire preimage in $\mathbb{Z} \llbracket t \rrbracket\left[x_{1}, \ldots, x_{4}\right]$.

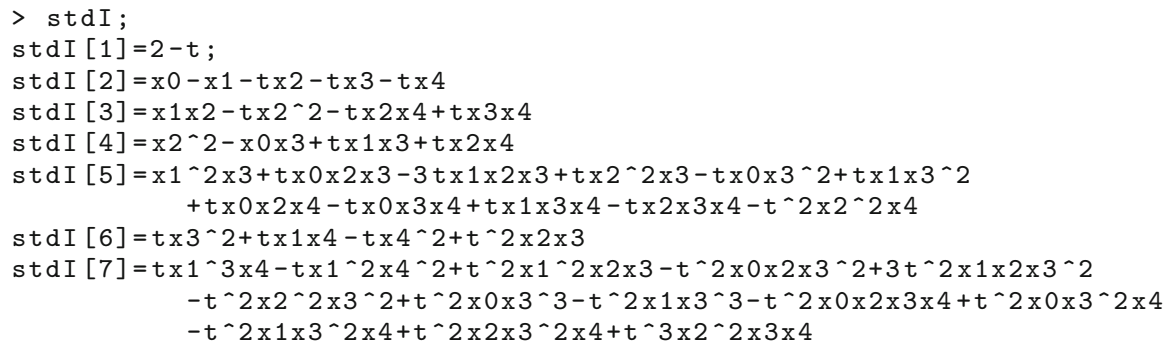

Fig. 6 Standard basis for computing a preimage SINGULAR

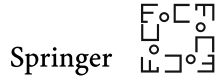


Acknowledgements Open access funding provided by Max Planck Society.

Open Access This article is distributed under the terms of the Creative Commons Attribution 4.0 International License (http://creativecommons.org/licenses/by/4.0/), which permits unrestricted use, distribution, and reproduction in any medium, provided you give appropriate credit to the original author(s) and the source, provide a link to the Creative Commons license, and indicate if changes were made.

\section{References}

1. X. Allamigeon, P. Benchimol, S. Gaubert, and M. Joswig, Combinatorial simplex algorithms can solve mean payoff games, SIAM J. Optim. 24, no. 4, 2096-2117 (2014).

2. E. Baldwin and P. Klemperer, Understanding Preferences: 'Demand Types', and The Existence of Equilibrium with Indivisibilities. Available at SSRN, https://doi.org/10.2139/ssrn.2643086 (2018)

3. Rouchdi Bahloul and Nobuki Takayama, Local Gröbnerfans, C. R. Math. Acad. Sci. Paris 344, no. 3, 147-152 (2007).

4. Janko Böhm, Wolfram Decker, Claus Fieker, Santiago Laplagne, and Gerhard Pfister, Bad primes in computational algebraic geometry, Mathematical Software - ICMS 2016, 93-101 (2016).

5. T. Bogart, A. N. Jensen, D. Speyer, B. Sturmfels, and R. R. Thomas, Computing tropical varieties, J. Symbolic Comput. 42, no. 1-2, 54-73 (2007).

6. Andrew Chan, Gröbner bases over fields with valuation and tropical curves by coordinate projections, Ph.D. thesis, University of Warwick (2013).

7. Andrew J. Chan and Diane Maclagan, Gröbner bases over fields with valuations, Math. Comput. 88, no. 315, 467-483 (2019).

8. Wolfram Decker, Gert-Martin Greuel, Gerhard Pfister, and Hans Schönemann, SINGULAR 4-1-2 - $A$ computer algebra system for polynomial computations, https://www.singular.uni-kl.de (2019).

9. Andreas Gathmann, Tropical algebraic geometry, Jahresber. Dtsch. Math.-Ver. 108, No. 1, 3-32 (2006).

10. Daniel R. Grayson and Michael E. Stillman, Macaulay2, a software system for research in algebraic geometry, https://www.math.uiuc.edu/Macaulay2/ (2019).

11. Walter Gubler, A guide to tropicalizations, Algebraic and combinatorial aspects of tropical geometry, Contemp. Math. 589, 125-189 (2013).

12. M. Hampton, and A. Jensen, Finiteness of spatial central configurations in the five-body problem, Celestial Mech. Dynam. Astronom. 109, no. 4, 321-332 (2011).

13. Tommy Hofmann, Yue Ren, Computing tropical points and tropical links, Discrete Comput. Geom. 60, no. 3, 627-645 (2018).

14. Anders N. Jensen, Gfan 0.6.2, a software system for Gröbner fans and tropical varieties, http://home. math.au.dk/jensen/software/gfan/gfan.html (2017).

15. Anders N. Jensen, Hannah Markwig, Thomas Markwig, and Yue Ren, tropical . 1 ib. a SINGULAR 4-1-2 library for computations in tropical goemetry (2019).

16. Anders N. Jensen, Yue Ren, and Frank Seelisch, g fan. 1ib. A SingulaR 4-1-2 interface to gfanlib for basic computations in convex geometry (2019).

17. Nikita Kalinin, Tropical approach to Nagata's conjecture in positive characteristic, Discrete Comput. Geom. 58, no. 1, 158-179 (2017).

18. Diane Maclagan and Bernd Sturmfels, Introduction to tropical geometry, Graduate Studies in Mathematics, vol. 161, American Mathematical Society, Providence, RI (2015).

19. Thomas Markwig, Yue Ren, and Oliver Wienand, Standard bases in mixed power series and polynomial rings over rings, J. Symbolic Comput. 79, 119-139 (2017).

20. Thomas Markwig and Yue Ren, Gröbnerfans of $x$-homogeneous ideals in $R \llbracket t \rrbracket[x]$, J. Symbolic Comput. 83, 315-341 (2017).

21. Hideyuki Matsumura, Commutative Algebra, 2nd ed., Mathematics Lecture Note Series, vol. 56, (1980).

22. G. Mikhalkin, Enumerative tropical algebraic geometry in $\mathbb{R}^{2}$, J. Amer. Math. Soc. 18, no. 2, 313-377 (2005)

23. T. Mora, and L. Robbiano, The Gröbner fan of an ideal, J. Symbolic Comput. 6, no. 2-3, 183-208 (1988). 
24. L. Pachter, and B. Sturmfels, Algebraic statistics for computational biology, Cambridge Univ. Press, New York (2005).

25. A. Popescu, Signature based standard bases over principal ideal rings, $\mathrm{PhD}$ Thesis, TU Kaiserslautern (2016).

26. Patrick Popescu-Pampu and Dmitry Stepanov, Local tropicalization, Algebraic and combinatorial aspects of tropical geometry, Contemp. Math., vol. 589, 253-316 (2013).

27. Y. Ren, Tropical geometry in SINGULAR, Dissertation, Technische Universität Kaiserslautern, Germany (2015).

28. Sage, Sagemath, the Sage Mathematics Software System (Version 8.7), https://www.sagemath.org (2019).

29. Jeff Sommars and Jan Verschelde, Pruning algorithms for pretropisms of Newton polytopes, Computer Algebra in Scientific Computing - CASC 2016, 489-503 (2016).

30. David Speyer and Bernd Sturmfels, The tropical Grassmannian, Adv. Geom. 4, no. 3, 389-411 (2004).

31. Bernd Sturmfels, Gröbner bases and convex polytopes, University Lecture Series, vol. 8, American Mathematical Society, Providence, RI (1996).

32. N. M. Tran, and J. Yu, Product-Mix Auctions and Tropical Geometry, arXiv:1505.05737 (2015).

33. Naoyuki Touda, Local tropical variety, arXiv:math/0511486 (2005).

34. Tristan Vaccon, Matrix-F5 algorithms over finite-precision complete discrete valuation fields, J. Symbolic Comput. 80, Part 2, 329-350 (2017).

Publisher's Note Springer Nature remains neutral with regard to jurisdictional claims in published maps and institutional affiliations. 